was used as method of analysis. Four main themes emerged from the data:

i. emotional landscapes,

ii. adaptation to a new situation (with subthemes: creating control, external and internal support in a difficult situation, normalizing the abnormal and reconciliation to uncertainty),

iii. moments of rebirth and

iv. transformation of attitudes towards life and the existence.

\section{CRANIAL NEUROSURGERY WITHOUT HAIR REMOVAL AND SHAMPOO CARE: RETROSPECTIVE ANALYSIS OF 450 CASES}

doi:10.1136/archdischild-2012-302724.0031

S Pinotti, S Vergna. Meyer Children Hospital, Florence, Italy

Background Trichotomy is a standard procedure in neurosurgery that aims at reducing infection rates. In children, psychological consequences associated to the whole head's hair removal are remarkable. Over the past twenty years the risk of infection associated to neurosurgical procedures has been thoroughly analyzed. Two negative consequences are associated to trichotomy: the loss of the natural defences offered by hair, and multiple skin lesions that increase bacterial growth. Our hospital established a protocol consisting of head washes before and after cranial neurosurgery with antiseptic shampoo, followed by daily washes with neutrum soap when the patient is discharged ("shampoo care").

Goal. To assess infection rate in a population of children who underwent cranial neurosurgery without hair removal.

Methods Retrospective analysis of clinical records of children undergoing cranial neurosurgery with "shampoo care" during one year.

Results 450 children were included. $5.55 \%$ of them had a wound complication, with only two cases of infection ( $0.4 \%)$. Available literature was revised to assess the effectiveness of our protocol. Wound complication and infections rates found in our experience were lower than those reported in patients treated with a standard trichotomy.

Discussion Cranial neurosurgery without hair removal and "shampoo care" is an effective method to reduce infection rates. This results in a shorter hospital stay, better self-esteem and improved quality of life when the child goes back to family life and school.

\section{CHRONIC PAIN IN THE NEWBORN, A DELPHI SURVEY TO DEFINE THE CONCEPT}

doi:10.1136/archdischild-2012-302724.0032

CJ van Ganzewinkel, P Andriessen. Neonatology, Maxima Medical Center, Veldhoven, The Netherlands

Chronic pain in the newborn has been poorly addressed in neonatal pain research. To date there is no clear definition. A three round, webbased Delphi survey aiming at providing a definition of chronic pain in the newborn was designed. We invited an international panel of experts in the field of neonatology and neonatal pain to participate.

Participants ( $\mathrm{n}=189)$ answered in the first round three openended questions: (1) what is the definition of chronic pain, (2) what are possible causes and (3) what are the signs and symptoms? The answers were categorized into 437 statements. These statements were valued by the participants on a 5 -point Likert scale in the second round. Statements with mode or median $\geq 4$ and mean $\geq 3.75$ were selected for analysis in the third round of the survey. These threshold values were used to provide the opportunity to reach consensus in subsequent rounds. In the third round the remaining participants $(n=72)$ were asked to reflect on the group response regarding the remaining 65 statements. Provided with their own value from the previous round, the participants were able to revalue the statements. This process resulted in 34 statements with mode, mean and median of $\geq 4$, in which the participants reached consensus.

Several etiologic factors were defined, but no useful diagnostic criterion could be identified. The Delphi survey resulted in a description of chronic pain in the newborn. Identifying chronic pain is clinically relevant because it interferes with growth, prolongs hospitalization and leads to altered pain perception.

\section{THE BLACK BOX OF PAIN ASSESSMENT IN EXTREMELY PREMATURE NEWBORNS REMAINS CLOSED}

doi:10.1136/archdischild-2012-302724.0033

M van Dijk, D Roofthooft, I Reiss. Neonatal Intensive Care, Erasmus MC - Sophia Children's Hospital, Rotterdam, The Netherlands

Background and aims In our NICU nurses assess the neonates' pain with the validated COMFORTneo scale three times per day and additionally if they suspect pain. We treat more and more extremely premature neonates from 24 weeks gestation. In this study we explore if the COMFORTneo scale is valid for these extreme premature neonates.

Methods COMFORTneo scores and Numeric Rating Scale (NRS) scores for pain and distress from 2011 were extracted from the patient data management system. We selected scores assigned in the first 28 postnatal days and considered three gestational age groups: extreme prematures: 23.6 to 27.0 wks, prematures: 27.1 to 35.6 weeks and term borns: 36 weeks and older.

Results We retrieved 9915 scores in 638 newborns. The median number of scores per patient was 41 (IOR 28 to 55) for 76 extreme prematures; 8(IOR 3 to 18) for 329 prematures; and 3 (IOR 1 to 9) for 233 term borns. $10.0 \%$ of scores for the extreme prematures; $9.1 \%$ of scores for the prematures; and $15.8 \%$ of scores for the term borns suggested pain or distress (COMFORTneo score $\geq 14$ ). Correlations between the COMFORTneo scale and the NRS pain ranged from 0.42 (extreme prematures) to 0.53 (prematures); those between the COMFORTneo scale and the NRS-distress 0.76 (extreme prematures) to 0.85 (term borns). Internal consistencies varied from Cronbach's alpha 0.73 (in extreme prematures) to 0.85 (in term borns).

Conclusions The COMFORTneo scale has acceptable psychometric properties for extreme prematures but we should continue to study other assessment strategies.

\section{PARENTAL VIEWS OF PAEDIATRIC INTENSIVE CARE} TRANSFERS

doi:10.1136/archdischild-2012-302724.0034

F Bickell. PICU, Guy's \& St Thomas' NHS Foundation Trust, London, UK

Background In 2001a study was performed exploring the parental experience of our combined retrieval service for critically ill children, (Colville, Orr \& Gracey 2003). The results of this study changed the way the service was provided and introduced the opportunity for a family member to travel with their child in the ambulance for the transfer. Ten years on it was decided to repeat a questionnaire to gain an insight into how families perceived the current service and seek further ways to improve the parental experience at this stressful time.

Method Having gained approval from the Research and Develop team at the base hospital a questionnaire was given to all families whose child was transferred by the South Thames Retrieval Service to the Evelina Children's Hospital during January 2012. 